\title{
Detection of Pork Gelatin in Jelly Candy Using Fourier Transform Infrared (FTIR) and Polymerase Chain Reaction (PCR)
}

\author{
Jariyah $^{1 *}$, Ratna Yulistiani ${ }^{1}$, Shelma Wharda Afdilah ${ }^{1}$, Kusuma Wardhani Mas'udah ${ }^{2}$ \\ ${ }^{1}$ Department of Food Technology, Faculty of Engineering, University of Pembangunan Nasional "Veteran" Jawa Timur, Indonesia \\ ${ }^{2}$ Department of Mechanical Engineering, Faculty of Engineering, University of Pembangunan Nasional "Veteran" Jawa Timur, \\ Indonesia
}

\begin{abstract}
This study aims to identify the content of pork gelatin in jelly candy using Fourier Transform Infrared (FTIR) and Polymerase Chain Reaction (PCR) methods. This method provide information to the public in choosing halal and tested food products. By uses a stepwise cluster survey method to obtain a sample then the samples obtained were isolated in gelatin, analyzed using the FTIR spectrophotometer method, and continued with data analysis using PCA (Principal Component Analysis). In addition, DNA detection analysis of pork gelatin was carried out using the PCR method. The results of the study were FTIR spectrum at wavelengths of $1450-1300 \mathrm{~cm}^{-1}, 1543 \mathrm{~cm}^{-1}$, and $2800-3000 \mathrm{~cm}^{-1}$. The classification of gelatin sources in jelly candy with PCA resulted in the proportion value of Principal Component 1 (PC 1) of $39 \%$, the value of the proportion of Principal Component 2 (PC 2) of $31 \%$, the value of the proportion of Principal Component 3 (PC 3) of $14.5 \%$ and the cumulative value of PC 1, PC 2, and PC 3 is $84.5 \%$. DNA amplification of jelly candy samples by PCR proved that all jelly candy samples A, B, C, D, and E did not contain pork.
\end{abstract}

Keywords: FTIR, PCR, Principal Component Analysis

\section{Introduction}

Halal food products in a country with a Muslim majority population is a major concern. Some of the ingredients used in food products such as gelatin, collagen, and fat are synthesized or extracted from mammals such as cattle, fish, and pigs. Products sourced from pork and its derivatives are prohibited for Muslims [1]. Meanwhile, the halal lifestyle is sweeping the world, not only in countries with Muslim-majority populations but also in countries with Muslim-minority populations. So the demand for halal products is quite large. Halal aspects are very broad, such as goods and/or services related to food, beverages, drugs, cosmetics, chemical products, biological products, genetically engineered products, as well as goods used, used, or utilized by the community [2].

Gelatin is a product in the form of hydrocolloids derived from the hydrolysis of animal or livestock collagen protein, which is hydrophilic which is found in many skins, bones, and animal tissues [3]. The use of gelatin as a gelling agent in jelly candy products needs to be considered because almost $80 \%$ of the gelatin produced comes from pork-skin. As explained by one of the gelatin supply companies, Gelatine Manufacturers of Europe (GME) that in 2018 almost $80 \%$ of gelatin produced came from pork skin, $15 \%$ came from split (thin layer on cowhide), while the remaining 5\% derived from beef, fish, and pork bones [4].

The National Standardization Agency (2008) stated that jelly candy is a soft textured confectionery, which is processed with the addition of hydrocolloid components such as agar, gum, pectin, starch, carrageenan, gelatin, and others. which is used for texture modification to produce a chewy product [5]. One component of the hydrocolloid forming jelly candy as much as $23 \%$ of the use of gelatin in the food industry of 154,000 tons. Jelly candy is made by cooking sugar until it reaches the desired solid, then adding gelling ingredients (gelatin, agar, pectin, and carrageenan) then adding flavor and color, and finally printing [6].

The jelly candy in circulation uses a thickening agent in the form of gelatin, so it requires an analytical method that can ensure that the gelatin used is free from pork content. Gelatin contained in jelly candy can be identified using FTIR (Fourier Transform Infrared) spectroscopy and FTIR infrared spectrum data processing using chemometric methods with Principal Component Analysis (PCA) techniques. PCA is one of the chemometric methods for classifying the properties of a material or substance based on the similarities it has [7]. Through PCA modeling, it can provide an overview

\footnotetext{
* Corresponding author : jariyah.tp@upnjatim.ac.id
} 
in classifying the profiles of bovine and pork gelatin [8], [9]. Furthermore, a confirmation method of porcine gelatin DNA detection analysis using Polymerase Chain Reaction (PCR) was used to support the results of this study. PCR is a technique for synthesis and amplification of DNA in vitro, through an enzymatic process using DNA polymerase enzymes and nucleotide primers that will hybridize with parts of DNA from two opposite directions [10]. The purpose of this study was to identify pork gelatin in jelly candy products in circulation using the Fourier Transform Infrared (FTIR) and Polymerase Chain Reaction (PCR) methods and to determine the source of gelatin used in jelly candy products.

Table 1. Percentage of transmittance at a wavelength of $1450-1300 \mathrm{~cm}^{-1}$

\begin{tabular}{|c|c|c|}
\hline Gelatin Sample & $\begin{array}{l}\text { Wavelength } \\
\left(\mathrm{cm}^{-1}\right)\end{array}$ & $\begin{array}{c}\text { Percentage } \\
\text { transmittance } \\
(\%)\end{array}$ \\
\hline Pig Control-1 & $\begin{array}{l}1450 \\
1384\end{array}$ & $\begin{array}{l}31 \\
31\end{array}$ \\
\hline Pig Control -2 & $\begin{array}{l}1441 \\
1384\end{array}$ & $\begin{array}{l}31 \\
31\end{array}$ \\
\hline Cow Control-1 & 1384 & 61 \\
\hline Cow Control-2 & 1384 & 31 \\
\hline Jelly candy B-1 & $\begin{array}{l}1419 \\
1383\end{array}$ & $\begin{array}{l}53 \\
52\end{array}$ \\
\hline Jelly candy B-2 & $\begin{array}{l}1419 \\
1383\end{array}$ & $\begin{array}{l}53 \\
52\end{array}$ \\
\hline Jelly candy C-1 & $\begin{array}{l}1420 \\
1376\end{array}$ & $\begin{array}{l}65 \\
65\end{array}$ \\
\hline Jelly candy E-1 & $\begin{array}{l}1366 \\
1341 \\
1318\end{array}$ & $\begin{array}{l}35 \\
36 \\
37\end{array}$ \\
\hline Jelly candy E-2 & 1378 & 49 \\
\hline
\end{tabular}

\section{Research Method}

\subsection{Research Materials}

The ingredients used in the analysis of pork gelatin in jelly candy with FTIR were jelly candy purchased at a traditional market in East Surabaya (Indonesia), 10\% acetic acid, saturated picric acid, $\mathrm{KBr}$ pellets, aquadest. Ingredients for DNA detection in pork gelatin include pork, jelly candy A, jelly candy B, jelly candy $C$, jelly candy $\mathrm{D}$, jelly candy E proteinase-K $31+\mathrm{TE}$ buffer 750 1 (TE pH 8 \& SDS $1 \%(\mathrm{v} / \mathrm{v})), 201 \mathrm{NaCl} 5 \mathrm{M}$, DNAZol $1 \mathrm{cc}$, phenol, chloroform, isopropanol, $70 \%$ ethanol, sterile distilled water. The materials used in DNA gelatin analysis were distilled water, DNA template, TE Buffer, dNTPs, $\mathrm{MgCl} 2$, Taq DNA polymerase, nuclease water, pork primer sequence with base sequence 5'-GCC TAA ATC TCC CCT CAA TGG TA-3' and 5'-ATG
AAA GAG GCA AAT AGA TTT TCG-3', Ethidium Bromide, $2 \%$ agarose $(0.4 \mathrm{gr}+20 \mathrm{ml}$ TBE $0.5 \mathrm{x})$.

\subsection{Research Tools}

The tools used for the extraction of gelatin jelly candy in this study were analytical scales, glassware, heater, thermometer, scissors, mortar, magnetic stirrer, oven, filter paper. The tools used in gelatin analysis include Shimadzu Perkin Elmer Fourier Transform Infrared (FTIR) spectrophotometer, Shimadzu hand press, Microsoft Windows XP Professional operating system computer, and Minitab 16 software for chemometric analysis. The tools used in DNA detection in gelatin are Applied Biosystems GeneAmp PCR System 9700, Mupid-2plus electrophoresis machine, analytical balance, microtube, cold centrifuge, water bath, vortex, oven, Eppendorf tube, heater, and glassware.

\subsection{Identification of Gelatin Using FTIR}

Identification of gelatin using FTIR spectrophotometer was carried out by dissolving $2 \mathrm{mg}$ of isolated gelatin mixed with $200 \mathrm{mg}$ of $\mathrm{KBr}$ and then homogenized. Pellets were formed using a hand press (Shimadzu) with a pressure of 8 tons for 10 minutes. FTIR spectrum measurements were carried out at $4000-400 \mathrm{~cm}^{-1}$. The results of the spectrum measurements will appear on a personal computer equipped with OPUS software version 4.2 [9].

\subsection{Identification of Gelatin Using the FTIR Method}

Identification of gelatin using FTIR spectrophotometer was carried out by dissolving $2 \mathrm{mg}$ of isolated gelatin mixed with $200 \mathrm{mg}$ of $\mathrm{KBr}$ and then homogenized. Pellets were formed using a hand press (Shimadzu) with a pressure of 8 tons for 10 minutes. FTIR spectrum measurements were carried out at $4000-400 \mathrm{~cm}^{-1}$. The results of the spectrum measurements will appear on a personal computer equipped with OPUS software version 4.2 [9].

\subsection{Identification of Pork Gelatin Using PCR}

\subsubsection{Extraction of DNA Gelatin in Jelly Candy}

Extraction and isolation of gelatin DNA in DNA jelly candy was carried out first by separating the non-DNA components which consisted of several steps. 2.5 grams of jelly candy sample was dissolved in $5 \mathrm{ml}$ of distilled water. It is then heated until the sample is completely dissolved. A total of 5001 of the sample was inserted into a $1.5 \mathrm{ml}$ microtube by adding 31 of proteinase- $\mathrm{K}$ and 7501 of TE buffer (Tris-EDTA pH 8 and SDS 1\% $(\mathrm{v} / \mathrm{v})$. The microtube containing the sample was then incubated in a water bath at a temperature of $55{ }^{\circ} \mathrm{C}$ for 16 hours, then centrifuged at $3000 \mathrm{rpm}$ for 10 minutes. The results of the centrifugation were then separated between the supernatant and the pellets formed. 
The supernatant obtained was transferred to a new microtube containing 201 of $5 \mathrm{M} \mathrm{NaCl}$. The supernatant was incubated at $37{ }^{\circ} \mathrm{C}$ for 1 hour. Next, it was centrifuged at $3000 \mathrm{rpm}$ for 10 minutes and the supernatant was separated from the pellets formed. The volume of the supernatant was measured. The supernatant was transferred back to a new microtube and phenol was added according to the volume of the supernatant (1:1), the sample was vortexed until homogeneous. $0.2 \mathrm{cc}$ of chloroform was added and then vortexed again. Samples were incubated for 24 hours. Samples were centrifuged at $6000 \mathrm{rpm}$ for 15 minutes, separated between the supernatant and the formed pellets.

Volume measurements were carried out on the obtained supernatant. The supernatant was transferred back to a new microtube and added isopropanol according to the volume of the supernatant $(1: 1)$, then the sample was incubated for 30 minutes. The sample was centrifuged again at $1200 \mathrm{rpm}$ for 10 minutes. The supernatant was separated from the formed pellet. The pellets were added with $0.5 \mathrm{cc}$ of $70 \%$ ethanol to the pellets and then vortexed until homogeneous. Samples were incubated for 2 hours and then centrifuged at $12000 \mathrm{rpm}$ for 10 minutes. The supernatant contained in the microtube was discarded, then the microtube was placed upside down for several minutes to dry. Add 501 of sterile distilled water to the pellets obtained and stored at $-20^{\circ} \mathrm{C}$. DNA from each sample was then tested for purity and concentration using spectrophotometry.

\subsubsection{Pork DNA Extract}

The pork DNA extraction method is a modification that refers to research (Al-Kahtani, Ismail, and Asif Ahmed, 2017) which uses the DNA isolation method Kit High Pure PCR Template (Roche, Mannheim, Germany) . However, in this study, DNA extraction and isolation methods were used using a DNAzol reagent kit. A total of 0.05 grams of pork samples were dissolved in $500 \mathrm{ml}$ of distilled water in a $15 \mathrm{ml}$ centrifugation tube. Samples were incubated for 24 hours at room temperature. $1 \mathrm{cc}$ of DNAZol was added to the sample and then vortexed until homogeneous. The samples were incubated again for 1 hour at room temperature. $0.2 \mathrm{cc}$ of chloroform was added to the sample and then vortexed for 2 minutes. Samples were incubated at room temperature for 24 hours and then centrifuged at $6000 \mathrm{rpm}$ for 15 minutes. Supernatant with pellets formed is separated [10].

The separated supernatant was then transferred to a new microtube and added isopropanol according to the volume of the supernatant (1:1). Samples were incubated for 30 minutes at room temperature. The sample was centrifuged again at $12000 \mathrm{rpm}$ for 10 minutes. Then the supernatant was removed and $0.5 \mathrm{cc}$ of $70 \%$ ethanol was added and vortexed until homogeneous. The sample was again incubated for 30 minutes and then centrifuged at $12000 \mathrm{rpm}$ for 10 minutes. The supernatant contained in the sample was discarded, then the microtube was placed upside down for several minutes to dry. $50 \mu 1$ of sterile distilled water was added to the pellet, then the pellet was stored at -20
${ }^{\circ} \mathrm{C}$. DNA from each sample was tested for purity and concentration using spectrophotometry.

\section{Result and Discussion}

\subsection{Peak of the Fourier Transform Infrared (FTIR) Spectrum of Gelatin}

The results of gelatin isolation of jelly candy samples B, $\mathrm{C}$, and $\mathrm{E}$ using the gelatin isolation method produced light yellow to dark yellow powdered gelatin solids [6]. The presence of yellow color in the isolated gelatin sample was due to the residual picric acid that was still present in the isolated gelatin. It is also found in research [11] that the use of picric acid in the extraction of gelatin in tablet drugs produces yellowish-colored powdered gelatin. The addition of picric acid which is a strong acid result in the presence of excess $\mathrm{H}+$ ions, which indicates the presence of turbidity. While jelly candy samples A and jelly candy samples D due to differences in composition, the gelatin was not isolated, this could be due to the low temperature used for isolation. Heating is carried out to dissolve the gelatin at least $49{ }^{\circ} \mathrm{C}$ or usually at a temperature of $60-70{ }^{\circ} \mathrm{C}[12]$. Therefore, due to the low isolation temperature, gelatin powder was not found in jelly candy A and jelly candy D. Jelly candies whose gelatin was isolated were then identified using FTIR.

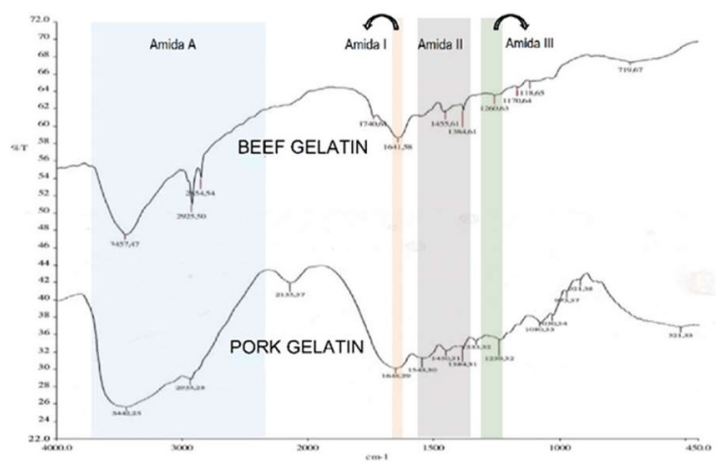

Fig. 1. Peak FTIR Spectrum of Cattle Gelatin Control and Pork Gelatin Control.

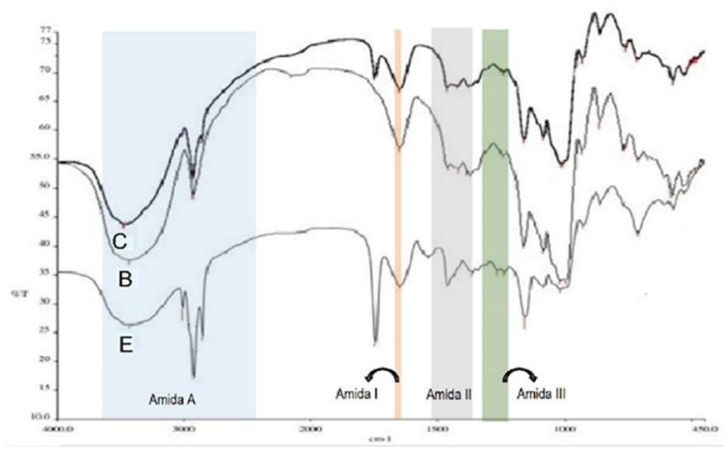

Fig. 2. Peak FTIR spectrum (a) gelatin jelly candy sample B, (b) gelatin jelly sample C, (c) gelatin jelly candy sample E.

Gelatin-like protein has a structure consisting of carbon, hydrogen, hydroxyl group $(\mathrm{OH})$, carbonyl group $(\mathrm{C}=\mathrm{O})$, and amine group $(\mathrm{NH})$ [9]. Figure 1. shows that 
the spectrum peaks found in the bovine gelatin control are 11 and the spectrum peaks found in the pork gelatin control are 14. Figure 2. Shows the peak spectrum in the jelly candy sample found with a total number of varying peaks, in jelly candy sample B, jelly candy sample C, and jelly candy sample $E$ have 23,21 , and 20 spectrum peaks, respectively. The number of peaks that vary is influenced by the vibration (vibration) of each molecule caused by the absorption of infrared light and will provide information in the form of the intensity of the infrared light absorbed, the more molecules that vibrate, the more diverse the peaks obtained. Infrared light passing through a compound causes vibrations between the molecules of the compound, resulting in a change in the dipole moment of a compound.

The typical wavelength of gelatin is divided into 4 parts, namely the absorption region of amide $\mathrm{A}$ at a wavelength of (3600-2300) $\mathrm{cm}^{-1}$, amide $\mathrm{I}$ at a wavelength (1636-1661) $\mathrm{cm}^{-1}$, amide II at a wavelength of (1560-1335). $\mathrm{cm}^{-1}$, and amide III at a wavelength $(1300-1200) \mathrm{cm}^{-1}$ [9]. Figure 26. shows that the results of the isolation of gelatin from samples of jelly candy B, jelly candy $\mathrm{C}$, and jelly candy $\mathrm{E}$ have all the typical absorption regions of gelatin in amide $\mathrm{A}$, amide I, amide II, and amide III according to the study [9]. Figure 26. shows that the results of the isolation of gelatin from samples of jelly candy B, jelly candy C, and jelly candy $\mathrm{E}$ have all the typical absorption regions of gelatin in amide A, amide I, amide II, and amide III according to the study [13]. So the isolation of gelatin in jelly candy samples was successfully carried out. Each typical wavelength of gelatin and its functional groups in jelly candy samples can be seen in Table 13 and Table 14 . Furthermore, the wavelengths used to distinguish the source of gelatin in jelly candy are in the region 1450 $1300 \mathrm{~cm}-1,1543 \mathrm{~cm}-1$, and 2800-3000 cm-1. Because in the region of $1450-1300 \mathrm{~cm}-1,1543 \mathrm{~cm}-1$, and $2800-$ $3000 \mathrm{~cm}-1$, there are differences in the amino acid composition of bovine and pork gelatin, especially for glycine, proline, and arginine [14]. This is in accordance with the amino acid composition of bovine and pork gelatin (as listed in Table 2) which shows the difference in residue composition per 1000 total amino acid residues of glycine, proline, and arginine types in bovine skin gelatin and pork skin gelatin ranging from 64 to 131.

Next will be discussed the wavelength in each of the above regions. The first region that can distinguish the source of gelatin is at a wavelength of $1450-1300 \mathrm{~cm}^{-1}$. All samples have a spectrum peak at a wavelength of $1450-1300 \mathrm{~cm}^{-1}$ which indicates the presence of the C$\mathrm{H}$ functional group. At a wavelength of $1450-1300 \mathrm{~cm}^{-1}$ indicates the presence of $\mathrm{C}-\mathrm{H}$. The wavelength region of $1450-1300 \mathrm{~cm}^{-1}$ can distinguish the source of gelatin because in this region the difference in amino acid composition between pork gelatin and beef gelatin can be seen through the percent transmittance (number of frequencies of infrared radiation that is not absorbed) of each sample. The smaller the percentage of transmittance obtained by the sample, the greater the infrared light absorbed by the compound [15]. The small percentage of transmittance obtained by the sample also indicates more compounds contained in the sample. The following is Table 9. percentage transmittance at wavelength $1450-1300 \mathrm{~cm}^{-1}$.

In table 1, the porcine control gelatin samples 1 and 2 have a transmittance percentage of 31 , which is lower than the bovine control gelatin samples 1 and 2, which are between 31-61. This is because the amino acid content in porcine gelatin is greater than that of beef gelatin so that infrared rays are absorbed more and produce a lower percent transmittance in porcine control gelatin. The smaller the percent transmittance means that almost all of the transmitted frequencies are absorbed by the compound. The thicker a layer or the greater the concentration, the more atoms there will be, as well as the more frequent collisions of light particles with atoms so that it is more difficult for light to pass through, the lower the percentage of transmittance obtained. It is also supported by the statement that the difference in frequency at a wavelength of 1450-1300 $\mathrm{cm}-1$ is indicated because the aliphatic amino acids of pork gelatin are greater than that of beef gelatin, where the amino acid composition between bovine gelatin and pork gelatin is relatively different, especially for glycine, proline and arginine residues [11].

However, the gelatin sample of jelly candy E-1 also found a small transmittance percentage between 35-37. This can be caused when the infrared ray is fired, there are still solvent compounds left in the gelatin sample of jelly candy E-1 which causes the compounds in jelly candy E-1 to be greater in addition to that the yellow color of gelatin isolated from jelly candy $\mathrm{E}$ is more concentrated than gelatin. On the other hand, the more Ag substrate coats the glass, the lower the transmittance percentage [5]. So the small percentage of transmittance obtained was due to the presence of residual solvent compounds in the gelatin jelly candy sample E-1 [16]. The second region that can distinguish the source of gelatin is at a wavelength of $1543 \mathrm{~cm}^{-1}$. Only the control sample of pork gelatin showed absorption at a wavelength of $1543 \mathrm{~cm}^{-1}$, while for jelly candy samples $\mathrm{B}, \mathrm{C}$, and $\mathrm{E}$ there was no absorption at a wavelength of $1543 \mathrm{~cm}^{-1}$. This is because the absorption of jelly candy samples B, C, and E has shifted to $1459,1461,1508$, 1542 , and $1536 \mathrm{~cm}^{-1}$.

This shift in the wave region is caused by the deformation of the $\mathrm{N}-\mathrm{H}$ and $\mathrm{C}-\mathrm{H}$ bonds in gelatin. The shift in wavenumber in amide II in the range of 1335$1560 \mathrm{~cm}^{-1}$ is due to the deformation of the $\mathrm{N}-\mathrm{H}$ bond while the wavelength at $1500-1200 \mathrm{~cm}^{-1}$ represents the deformation of $\mathrm{CH}_{2}$ [11]. Deformation of $\mathrm{NH}$ and $\mathrm{CH}$ bonds in gelatin is due to the triple helix structure of collagen which changes due to temperature and extraction time in the gelatin making process. The triple helix structure of collagen that has been heated or has been extracted into gelatin breaks down into an -helix structure. The $\alpha$-helical structure of gelatin can decompose again into coil bonds (random) when exposed to high temperatures in the gelatin isolation process in jelly candy and cause deformation of NH. In the $1335-1560 \mathrm{~cm}^{-1}$ region, there are abnormalities in the molecular structure due to the transformation of -helices into random coil structures due to the extraction process which is characterized by the occurrence of $\mathrm{N}-\mathrm{H}$ bending vibrations coupled with $\mathrm{C}-\mathrm{N}$ stretching 
vibrations [17]. Temperature and length of extraction time can cause triple helix changes as a result of the denaturation of collagen to gelatin [18]. Then the shift in wavelength in jelly candy samples B, C, and E 1459 , $1461,1508,1542$, and $1536 \mathrm{~cm}^{-1}$ was caused by the temperature and extraction time during gelatin manufacture. The third region that can distinguish the source of gelatin is the wavelength of $2800-3000 \mathrm{~cm}^{-1}$. All gelatin jelly candy samples have different spectral peaks at a wavelength of $2800-3000 \mathrm{~cm}^{-1}$ can be seen in Table 2. The wavelength of $3000-2850 \mathrm{~cm}^{-1}$ is the absorption region of the $\mathrm{C}-\mathrm{H}$ bond [12]. The following are the wavelengths and functional groups of the FTIR spectra at a wavelength of $2800-3000 \mathrm{~cm}-1$ can be seen in Table 2.

Table 2. Wavelength and functional groups FTIR spectra at a wavelength of $2800-3000 \mathrm{~cm}^{-1}$.

\begin{tabular}{|c|c|c|c|}
\hline Sample & $\begin{array}{l}\text { Wavelength } \\
\left(\mathrm{cm}^{-1}\right)\end{array}$ & Bonding & Function Group \\
\hline $\begin{array}{l}\text { Beef } \\
\text { gelatin } \\
\text { control }\end{array}$ & $\begin{array}{l}2925 \\
2854\end{array}$ & $\begin{array}{l}\mathrm{C}-\mathrm{H} \\
\mathrm{C}-\mathrm{H}\end{array}$ & $\begin{array}{c}\text { Alkana (strong) } \\
\text { Alkana } \\
\text { (bending) }\end{array}$ \\
\hline $\begin{array}{l}\text { Pork } \\
\text { gelatin } \\
\text { control }\end{array}$ & $\begin{array}{l}2935 \\
2933\end{array}$ & $\begin{array}{l}\text { C-H } \\
\text { C-H }\end{array}$ & $\begin{array}{l}\text { Alkana } \\
\text { Alkana }\end{array}$ \\
\hline $\begin{array}{c}\text { Jelly } \\
\text { candy } \\
\text { sample B }\end{array}$ & 2929 & $\mathrm{C}-\mathrm{H}$ & Alkana \\
\hline $\begin{array}{c}\text { Jelly } \\
\text { candy } \\
\text { sample C }\end{array}$ & $\begin{array}{l}2926 \\
2925 \\
2854 \\
\end{array}$ & $\begin{array}{l}\mathrm{C}-\mathrm{H} \\
\mathrm{C}-\mathrm{H} \\
\mathrm{C}-\mathrm{H} \\
\end{array}$ & $\begin{array}{l}\text { Alkana } \\
\text { Alkana } \\
\text { Alkana } \\
\end{array}$ \\
\hline $\begin{array}{c}\text { Jelly } \\
\text { candy } \\
\text { sample E }\end{array}$ & $\begin{array}{l}2925 \\
2854\end{array}$ & $\begin{array}{l}\text { C-H } \\
\text { C-H }\end{array}$ & $\begin{array}{l}\text { Alkana (strong) } \\
\text { Alkana (strong) }\end{array}$ \\
\hline
\end{tabular}

The results of the identification of wavelengths using FTIR can be concluded that the results of the isolation of jelly candy that have been studied are gelatin. Each jelly candy isolate has an absorption at a wavelength that can be used as a reference to continue the analysis using the Principal Component Analysis (PCA) method to determine the source of the gelatin used. Because the working principle of FTIR is to recognize the functional group of a compound from the infrared absorption carried out on the compound [9]. To find out more clearly the source of gelatin used in the jelly candy sample, further analysis was carried out at wavelengths of $1450-1300 \mathrm{~cm}^{-1}, 1543 \mathrm{~cm}^{-1}$, and $2800-3000 \mathrm{~cm}^{-1}$ using the Principal Component Analysis (PCA) method on the control sample. cattle, control pigs, sample B, sample $\mathrm{C}$, and sample $\mathrm{E}$.

\subsection{Analysis of PCA Data Processing Results}

The results of the wavenumber and absorbance analysis of the FTIR were analyzed using the Principal Component Analysis method. Each sample analyzed using FTIR has almost similar absorption peaks [9]. However, if it is observed quantitatively, there is a difference in the height of the peak due to differences in the absorbance value in each sample. The absorbance value of each absorption in each sample can be seen in Table 1 and Table 2. The difference in absorbance values is analyzed by comparing the spectral peak's FTIR. Therefore, the PCA method is used to group the data, so that the differences in each sample can be specifically identified [15]. Absorbance analysis was carried out in the region of $1450-1300 \mathrm{~cm}^{-1}, 1543 \mathrm{~cm}^{-1}$, and $2800-3000 \mathrm{~cm}^{-1}$ which will be processed by PCA using Minitab 16 software. The following are the wavelength regions $1450-1300 \mathrm{~cm}^{-1}, 1543 \mathrm{~cm}^{-1}$, and $2800-3000 \mathrm{~cm}^{-1}$ can be seen in Figure 3.

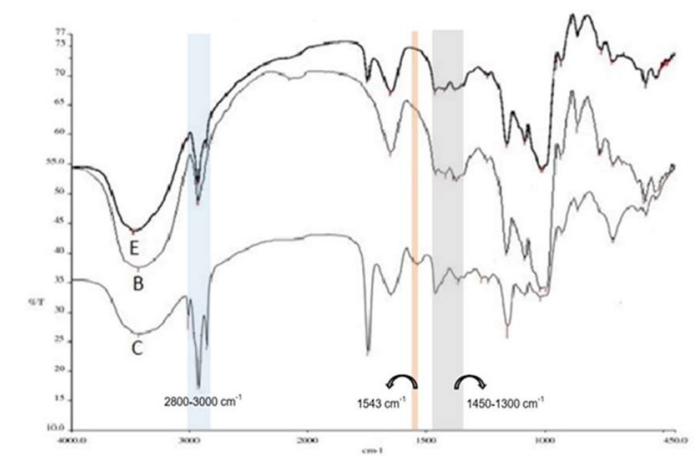

Fig. 3. The wavelength region is $1450-1300 \mathrm{~cm}^{-1}, 1543 \mathrm{~cm}^{1}$, and $2800-3000 \mathrm{~cm}^{-1}$.

The graphic results of the infrared spectrum pattern of absorption at wavelengths $1450-1300 \mathrm{~cm}-1,1543 \mathrm{~cm}-$ 1 , and 2800-3000 cm-1 can show differences in amino acid composition in beef and pork gelatin, especially for glycine, proline, and arginine. Principal Component (PC) plays a role in reducing the dimensions of the original variable so that a new variable (PC) is obtained which is not correlated with each other but stores some of the information contained in the original variable [14]. One way to find out how many PCs should be taken to reduce a variable is by using a scree plot or eigenvalue. The method used to determine the number of principal components (PC) is by looking at the eigenvalues that are more than one or observing the scree plot by looking at the angled fault of the scree plot. The following is a scree plot of the peak of the functional group obtained from the analysis results, which can be seen in Figure 4.

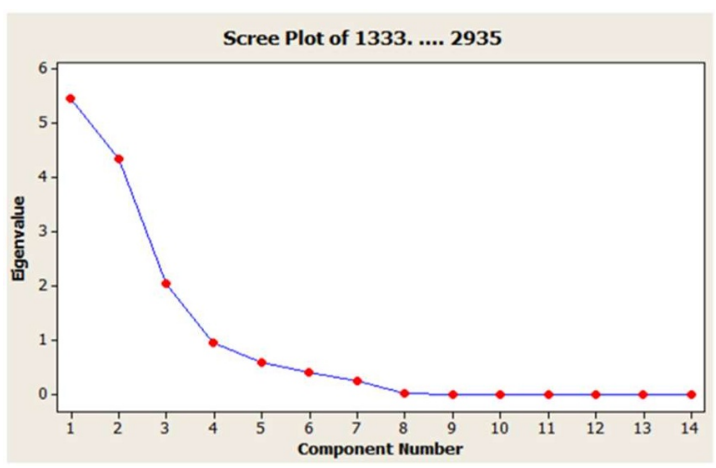

Fig. 4. Scree Plot Peak Functional Group 
Table 3. Correlation Matrix Eigen Analysis Table

\begin{tabular}{|c|c|c|c|}
\hline PC & Eigenvalues & Proportion & Cumulative \\
\hline 1 & 5.4545 & 0.390 & 0.390 \\
\hline 2 & 4.3438 & 0.310 & 0.700 \\
\hline 3 & 2.0297 & 0.145 & 0.845 \\
\hline 4 & 0.9399 & 0.067 & 0.912 \\
\hline 5 & 0.5800 & 0.041 & 0.953 \\
\hline 6 & 0.3969 & 0.028 & 0.982 \\
\hline 7 & 0.2523 & 0.018 & 1.000 \\
\hline 8 & 0.0028 & 0.000 & 1.000 \\
\hline 9 & 0.0000 & 0.000 & 1.000 \\
\hline 10 & 0.0000 & 0.000 & 1.000 \\
\hline 11 & 0.0000 & 0.000 & 1.000 \\
\hline 12 & 0.0000 & 0.000 & 1.000 \\
\hline 13 & 0.0000 & 0.000 & 1.000 \\
\hline 14 & 0.0000 & 0.000 & 1.000 \\
\hline
\end{tabular}

Based on Figure 4, the scree plot curve begins to slope or fracture at point 4 . This shows that there are three main components that can be taken, namely PC1, PC2, PC3. In addition, taking the number of PCs can be seen in the eigenvalues in Table 11. Eigenvalues greater than 1 are PC1, PC2, PC3. So it can be concluded that the number of PCs formed is three PCs. From Table 3. it can be seen that the proportional value of $\mathrm{PC} 1$ is $39 \%$, PC2 is $31 \%$, and PC3 is $14.5 \%$ so that the cumulative diversity of the proportion values is $84.5 \%$. This shows that the number of PCs selected can be used to distinguish the source of gelatin, the number of PCs selected is sufficient if it has a cumulative diversity of not less than $75 \%$ [19]. Furthermore, there is a score plot in the PCA analysis aimed at describing the data plot of the sample classification used. The following is a sample PCA score plot which can be seen in Figure 5 . The score plot displayed is a 2-dimensional plot in which there are 4 quadrants. The $\mathrm{x}$-coordinate is PC1/First Component and the y-coordinate is PC2/Second Component. In Figure 29 the area is divided into 4 quadrants, namely quadrant I, quadrant II, quadrant III, and quadrant IV.

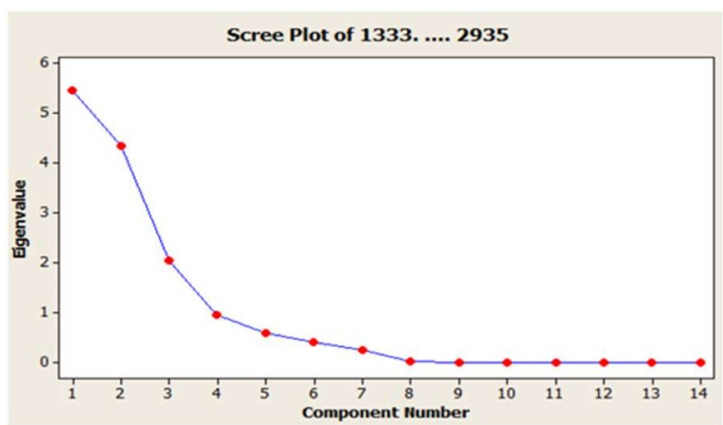

Fig. 5. Score Plot PCA Sample

Beef gelatin and gelatin isolated from jelly candy $\mathrm{C}$ and $\mathrm{E}$ are in quadrant II, pork gelatin is in quadrant III, and gelatin isolated from jelly candy B is in quadrant IV. Pork gelatin is in quadrant III, beef gelatin is in quadrant
II, and gelatin extracted from gummy is in quadrant II and IV. The PCA plot scores obtained showed that none of the samples were in the pig quadrant [14]. The closer the distance between the points, the closer the relationship possessed by the analyzed samples [5]. So it can be concluded that the gelatin jelly candy sample $\mathrm{B}$, sample $\mathrm{C}$, and sample $\mathrm{E}$ are free from pork gelatin content.

\subsection{Purity of DNA Isolate}

The DNA from each sample and control analyzed yielded the purity and concentration as shown in Table 4. The results of the concentration and purity of DNA isolation in jelly candy samples can be seen in Table 4 .

Table 4. Results of concentration and purity of DNA isolation on jelly candy samples.

\begin{tabular}{|c|c|c|}
\hline Sample & Concentration $(\mathrm{ng} / \mu l)$ & Purity \\
\hline Bk & 871.5 & 1.23 \\
\hline A & 539.0 & 1.25 \\
\hline B & 556.5 & 1.25 \\
\hline C & 479.5 & 1.26 \\
\hline D & 248.5 & 1.34 \\
\hline E & 633.5 & 1.27 \\
\hline
\end{tabular}

Note:

$\mathrm{Bk}=$ positive control pork

A-E = Sample jelly candy

The concentrations obtained ranged from 248.5 to $871,5 \mathrm{ng} / \mu l$, the pork DNA isolate concentration was $871,5 \mathrm{ng} / \mu l$; sample A was $539 \mathrm{ng} / \mu l$; sample B was $556,6 \mathrm{ng} / \mu \mathrm{l}$; sample $\mathrm{C}$ was $479,5 \mathrm{ng} / \mu \mathrm{l}$; sample D was $248,5 \mathrm{ng} / \mu l$; and sample E was $633,5 \mathrm{ng} / \mu l$. DNA with good quality generally has a concentration above 125 $\mathrm{ng} / \mu l[7]$. The results showed that the concentration obtained from DNA isolates had good quality because they were above $125 \mathrm{ng} / \mu \mathrm{l}$. With several levels above the DNA, the isolate can be continued for the PCR amplification process because PCR is quite sensitive and can detect levels up to $1 \mathrm{ng} / \mathrm{ml}$ [6].

The purity obtained from the control sample and the jelly candy sample ranged from 1.23 to 1.34 , the purity of the pork DNA isolate was 1.23 ; sample A is 1.25 ; sample $B$ is 1.25 ; sample $C$ is 1.26 ; sample $D$ is 1.34 ; and sample $\mathrm{E}$ is 1.27 . The value of DNA purity obtained was below 1.8, indicating that the isolated DNA still contained contamination in the form of the protein [10]. In general, with the purity value obtained, DNA extracted from all samples can still be used for the amplification process.

\subsection{Amplification Results with Polymerase Chain Reaction (PCR)}

The next process is the identification of pork content in jelly candy samples using PCR. The following is a visualization of PCR results from positive control pigs, jelly candy samples A, jelly candy samples B, jelly candy samples $C$, jelly candy samples $D$, and jelly candy samples E can be seen in Figure 6. 


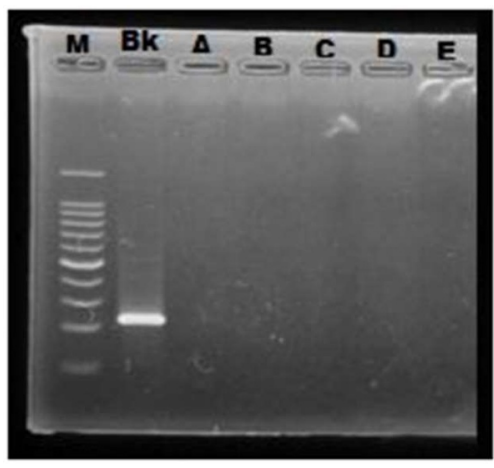

Fig. 6. Visualization of PCR results from each jelly candy, M $=100 \mathrm{bp}$ marker; $\mathrm{Bk}=$ pork control; $\mathrm{A}-\mathrm{E}=$ jelly candy sample.

The success of a PCR process depends on the primer used. The primer functions as a barrier for the target DNA fragment to be amplified and at the same time provides a hydroxy group $(\mathrm{OH})$ at the 3 ' end which is needed for the existence of DNA [10]. This study used pork primer with nucleotide base sequence 5'-GCC TAA ATC TCC CCT CAA TGG TA-3' and 5'-ATG AAA GAG GCA AAT AGA TTT TCG-3'. Based on the results of PCR visualization using $2 \%$ agarose gel electrophoresis in Figure 6. Shows the results of PCR amplification of cty $b$ from pork DNA as expected, which is about $212 \mathrm{bp}$, cty $\mathrm{b}$ gene amplicon from pork produces fragments of about 212 bp [14]. However, from the five samples of jelly candy that were tested, there was no indication that they contained/ contaminated pork DNA because there was no DNA band/band that appeared at $212 \mathrm{bp}$. The results of the gummy sample isolates did not show any DNA bands [10]. However, this does not show that the DNA isolation process was not successful because the isolation of pork DNA was successful. Meatball samples numbered 13 and pork DNA were amplified in the PCR results, while meatball samples numbered 1-12 were not amplified so it was concluded that samples numbered 112 did not contain pork. Based on the results of this PCR, it can be concluded that all of the jelly candy samples in circulation did not contain or were contaminated with pork or used beef gelatin.

\section{Conclusion}

There are 5 different brands of jelly candy products with a gelatin composition that are sold in the market. Two trademarks of jelly candy products that are labeled halal and 3 trademarks of jelly candy products that have not been labeled halal with gelatin composition. Identification of pork gelatin in jelly candy products was successfully carried out using a combination method of Fourier Transform Infrared (FTIR) and PCA, from samples of beef gelatin, pork gelatin samples, jelly candy samples B, jelly candy samples $C$, and jelly candy samples E. wavelengths $1450-1300 \mathrm{~cm}^{-1}, 1543 \mathrm{~cm}^{-1}$, and $2800-3000 \mathrm{~cm}^{-1}$. There are no jelly candy samples in the pork gelatin sample quadrant, so the gelatin used in the jelly candy samples does not contain pork gelatin, but contains beef gelatin. The results of the identification of FTIR and PCA were supported by the results of PCR research which showed that jelly candy products were detected negative for pork gelatin content because there was no DNA band that appeared at $212 \mathrm{bp}$.

We would like to thank the Faculty of Engineering, University of Pembangunan Nasional "Veteran" East Java, which has helped a lot in this research. So we can make this article.

\section{References}

1. L. Berti, R. D. Yetti, and R. Asra, "Chemical Analysis Methods to Detect Pork DNA and Derivative Contents in Food: A Review," Asian J. Pharm. Res. Dev., vol. 8, no. 9, pp. 10-19, (2020).

2. H. I. Abdullah Amqizal, H. A. Al-Kahtani, E. A. Ismail, K. Hayat, and I. Jaswir, "Identification and verification of porcine DNA in commercial gelatin and gelatin containing processed foods," Food Control, vol. 78, pp. 297-303, (2017), doi: https://doi.org/10.1016/j.foodcont.2017.02.024.

3. M. Muñoz-Colmenero, J. L. Martínez, A. Roca, and E. Garcia-Vazquez, "Detection of Different DNA Animal Species in Commercial Candy Products," J. Food Sci., vol. 81, no. 3, pp. T801T809, (2016), doi: https://doi.org/10.1111/17503841.13225.

4. Z. M. Zin, N. M. Sarbon, M. K. Zainol, S. N. Jaafar, M. M. Shukri, and A. H. A. Rahman, "Halal and Non-Halal Gelatine as a Potential Animal ByProducts in Food Systems: Prospects and Challenges for Muslim Community," Proc. First Int. Conf. Sci. Technol. Eng. Ind. Revolut. (ICSTEIR 2020), vol. 536, no. Icsteir 2020, pp. 530-540, (2021), doi: 10.2991/assehr.k.210312.086.

5. C. Cedeño-Pinos, M. Martínez-Tomé, M. A. Murcia, M. J. Jordán, and S. Bañón, “Assessment of rosemary (Rosmarinus officinalis L.) extract as antioxidant in jelly candies made with fructan fibres and Stevia," Antioxidants, vol. 9, no. 12, pp. 1-16, (2020), doi: 10.3390/antiox9121289.

6. S. Omar, M. Hasan, S. Abu-Romman, H. Ramadan, A. A. Qatatsheh, and H. Al-Dmoor, "Design and validation of short-amplicon length PCR assay for the detection of porcine gelatin in commercial candy and marshmallow products," Curr. Res. Nutr. Food Sci., vol. 6, no. 3, pp. 742747, (2018), doi: 10.12944/CRNFSJ.6.3.16.

7. M. Muñoz-Colmenero, J. L. Martínez, A. Roca, and E. García-Vázquez, "Authentication of commercial candy ingredients using DNA PCRcloning methodology.," J. Sci. Food Agric., vol. 96, no. 3, pp. 859-867, Feb. (2016), doi: $10.1002 /$ jsfa. 7158 .

8. H. Zhang et al., "Identification of Edible Gelatin Origins by Data Fusion of NIRS, Fluorescence Spectroscopy, and LIBS," Food Anal. Methods, vol. 14, no. 3, pp. 525-536, (2021), doi: 10.1007/s12161-020-01893-2.

9. R. Akbarzadegan, H. Ahari, A. Sharifan, and A. A. Anvar, "Overview of the studies on authentication of gelatin using Fourier Transform Infrared spectroscopy coupled with chemometrics," J. 
Human, Heal. Halal Metrics, vol. 1, no. 2, pp. 6471, (2020).

10. H. A. Al-Kahtani, E. A. Ismail, and M. Asif Ahmed, "Pork detection in binary meat mixtures and some commercial food products using conventional and real-time PCR techniques," Food Chem., vol. 219, pp. 54-60, (2017), doi: 10.1016/j.foodchem.2016.09.108.

11. S. Hermanto, L. O. Sumarlin, and W. Fatimah, "Differentiation of Bovine and Porcine Gelatin Based on Spectroscopic and Electrophoretic Analysis," J.Food Pharm.Sci, vol. 1, no. 1, pp. 6873, (2013), doi: 10.1021/ac60072a021.

12. P. Loukopoulos et al., "Thermal and structural study of drying method effect in high amylose starch- beta-carotene nanoparticles prepared with cold gelatinization," Carbohydr. Polym. Technol. Appl., vol. 2, no. May, p. 100092, (2021), doi: 10.1016/j.carpta.2021.100092.

13. Y. Liu, W. Pei, S. Sun, Q. Zhou, J. Wang, and J. Chen, "Protein-sugar interaction between Asini Corii Colla (donkey-hide gelatin) and Lycii Fructus (goji berry) evaluated by temperatureresolved ATR-FTIR and moving-window twodimensional correlation spectroscopy," J. Mol. Struct., vol. 1211, p. 128072, (2020), doi: 10.1016/j.molstruc. 2020.128072 .

14. N. Hassan, T. Ahmad, and N. M. Zain, "Chemical and Chemometric Methods for Halal Authentication of Gelatin: An Overview," J. Food Sci., vol. 83, no. 12, pp. 2903-2911, (2018), doi: 10.1111/1750-3841.14370.

15. N. Cebi, C. E. Dogan, A. E. Mese, D. Ozdemir, M. Arıc1, and O. Sagdic, "A rapid ATR-FTIR spectroscopic method for classification of gelatin gummy candies in relation to the gelatin source," Food Chem., vol. 277, pp. 373-381, (2019), doi: $10.1016 /$ j.foodchem.2018.10.125.

16. L. Wang et al., "Synergistic effect of highly aligned bacterial cellulose/gelatin membranes and electrical stimulation on directional cell migration for accelerated wound healing," Chem. Eng. J., vol. 424, no. March, p. 130563, (2021), doi: 10.1016/j.cej.2021.130563.

17. R. Akbarzadegan, H. Ahari, A. Sharifan, and A. A. Anvar, "Overview of the studies on authentication of gelatin using Fourier Transform Infrared spectroscopy coupled with chemometrics," $J$. Human, Heal. Halal Metrics, vol. 1, no. 2, pp. 6471, (2020).

18. N. Hassan, T. Ahmad, N. M. Zain, and A. Ashaari, "A novel chemometrics method for halalauthentication of gelatin in food products," Sains Malaysiana, vol. 49, no. 9, pp. 2083-2089, (2020), doi: 10.17576/jsm-2020-4909-06.

19. A. P. Roswiem and D. N. Mustaqimah, "Identification of Gelatin source in Toothpaste products using Combination of Attenuated Total Reflection-Fourier Transform Infrared ( ATRFTIR ) Spectroscopy and Chemometrics," Int. J. Halal Res., vol. 2, no. 1, pp. 30-39, (2020). 